\title{
Concepciones de los Docentes sobre la Evaluación Socialmente Justa
}

\author{
F. Javier Murillo $\left(^{*}\right)$ y Nina Hidalgo $\left(^{*}\right)$ \\ (*) Universidad Autónoma de Madrid - España
}

\section{RESUMEN}

El presente estudio busca conocer las concepciones de docentes sobre una evaluación socialmente justa. Para alcanzar dicho objetivo, se ha llevado a cabo una investigación fenomenográfica en la que han participado 30 docentes de educación Primaria y Secundaria, de centros públicos y privados y situados en contextos socioeconómicos favorables y desafiantes. Los resultados obtenidos muestran que los docentes conciben una evaluación socialmente justa conformada por seis elementos: una evaluación para el aprendizaje (más que del aprendizaje), equitativa y diferenciada para cada estudiante, participativa, que contribuya a la toma de conciencia social, que valore la autoestima y la empatía, y que favorezca y considere la capacidad reflexiva y critica de los estudiantes. Los hallazgos del estudio ponen de manifiesto la importancia de profundizar en concepciones sobre una evaluación socialmente justa del alumnado ya que la práctica evaluativa tiene claras repercusiones en la vida social y académica de los estudiantes.

Palabras Clave: Evaluación de estudiantes, Evaluación socialmente justa, Concepciones de evaluación, Investigación fenomenográfica.

\section{Teachers' Conceptions about a Socially Just Assessment}

\section{ABSTRACT}

The present study seeks to know assessment teachers' conceptions about what is a socially just assessment. To achieve this objective, a phenomenographical research has been carried out in which 30 teachers of Primary and Secondary education have participated, from public and private schools located in favourable and challenging contexts. The results obtained show how teachers conceive a socially just assessment consisting of six elements: assessment for learning (rather than learning), equitable and differentiated for each student, that contributes to social awareness, which value self-esteem and empathy, and that favor and consider the reflective and critical capacity of students. The findings of our study show the importance of deepening into conceptions about a socially just assessment of students since assessment practice has clear repercussions in social and academic students' life.

Keywords: Students assessment, Socially just assessment, Assessment conceptions, Phenomenographic research.

\section{Introducción}

Aunque la finalidad última de la Educación es el desarrollo integral de los estudiantes, cada vez más se le exige una mayor implicación en la construcción de sociedades más justas, equitativas e inclusivas. Contribuir a la formación de niños, niñas y adolescentes como agentes de cambio social se constituye, de esta manera, como un objetivo prioritario de los centros educativos.

La evaluación de los estudiantes, elemento fundamental de los procesos de enseñanza y aprendizaje, no puede estar ajena a esa exigencia. Efectivamente, la evaluación marca la forma de enseñar por parte del docente e incide en los comportamientos y actitudes de sus estudiantes (Murillo e Hidalgo, 2015a). Si se quiere una educación que de verdad contribuya a crear una sociedad más justa, se necesita una evaluación que trabaje en esa misma dirección: una evaluación socialmente justa. Sin embar- go, no hay acuerdo en qué significa una evaluación socialmente justa. Desde luego, para que una evaluación sea socialmente justa debe ser justa, pero no solo eso, también debe contribuir a generar en el estudiante conciencia social y fomentar su sentido crítico, creativo y constructivo. Como antes se señaló, ha de contribuir a formar estudiantes como agentes de cambio social.

Las concepciones de los docentes son el paso previo a la actuación, por lo que es importante conocer qué consideran los docentes que es una evaluación socialmente justa. En esa idea, en los últimos años se han desarrollado algunas investigaciones que buscan conocer las concepciones de los docentes sobre una evaluación justa (p.ej. Murillo e Hidalgo, 2017; Scott, Webberb, Lupartc, Aitkend y Scott, 2014, entre otros). Sin embargo, existen muy pocos trabajos que busquen profundizar en qué es una evaluación socialmente justa y los que existen lo hacen de forma teórica y no empírica (McArthur, 2015). 


\section{Marco Teórico}

Se entiende por concepción un sistema organizado de creencias originadas en las experiencias del individuo y desarrolladas a través de las distintas interacciones que tiene la persona con su entorno (Coll y Remesal, 2009; Remesal, 2011). Las concepciones tienen un carácter marcadamente social dado que se construyen y fundamentan en espacios compartidos con otras personas (Van den Berg, 2002). Aplicadas al ámbito educativo, las concepciones de los docentes pueden definirse como la estructura de creencias y vivencias construidas en un contexto social que tienen los profesores y que determinan la práctica educativa y los procesos de enseñanza y aprendizaje (Prieto y Contreras, 2008; Thompson, 1990).

Precisamente por la importancia de las concepciones en los procesos de enseñanza-aprendizaje, en los últimos años se han multiplicado las investigaciones que buscan conocer las concepciones de los docentes, así como de estudiantes y otros colectivos. En una investigación realizada hace ya 25 años, el profesor Frank Pajares (1992) afirmó que profundizar en las concepciones del profesorado debería ser un tema prioritario en la investigación educativa. En diversos estudios que desarrolló, concluyó que las ideas implícitas del profesorado siempre determinan su práctica en el aula, por lo que, si queremos cambiar el proceso de enseñanza-aprendizaje es fundamental conocer en profundidad dichas concepciones.

Dentro de la línea de las concepciones de los docentes cobran especial relevancia las investigaciones que se centran en conocer dichas concepciones acerca de la evaluación (Atjonen, 2014; Brown, 2004; Brown y Hirschfeld, 2008; Brown y Remesal, 2012; Hidalgo y Murillo, 2017; Remesal, 2011; Winterbottom, Brindley, Taber, Fisher, Finney y Riga, 2008). De acuerdo con Prieto (2008), la influencia de las concepciones en la evaluación que llevan a cabo los docentes es clara: las creencias que tiene el profesorado tienen repercusiones directas sobre su práctica evaluativa y éstas en el aprendizaje de los estudiantes.

En los últimos años, la investigación sobre concepciones de los docentes ha prestado un especial interés en las concepciones acerca de una evaluación justa de los estudiantes (Pettifor y Saklofske, 2012; Sambell, McDowell y Brown, 1997; Suskie, 2002). De estos trabajos se concluye que hay dos grandes concepciones: aquellas relacionadas con una evaluación justa igualitaria (Lam, 1995) y otras que vinculan la justica con la equidad ( $\mathrm{Pe}-$ pper y Pathak, 2008, Pettifor y Saklofske, 2012; Scott et al., 2014; Suskie, 2002; Tierney, Simon y Charland, 2011; Stobart, 2005). En el cuadro 1 se han resumido las características de cada una de ellas en cuando a los métodos, momentos, instrumentos y uso de los resultados de la evaluación.

Cuadro 1. Diferencias entre las concepciones sobre una evaluación justa igualitaria y equitativa

\begin{tabular}{ll}
\hline \multicolumn{1}{c}{ Evaluación JUSTA IGUAlitaria } & \multicolumn{1}{c}{ Evaluación JUSTA EQUitativa } \\
\hline Criterios externos & Adaptar los criterios según las necesidades de cada estudiante \\
Objetividad & Subjetividad a favor de cada estudiante \\
Transparencia y explicitación de los criterios & Individualización de los criterios de evaluación \\
Rendimiento & Progreso y esfuerzo \\
Examen como elemento clave de evaluación & Restar peso al examen en la evaluación \\
Uso de pruebas cualitativas solo como complemento del examen & Uso de pruebas cualitativas de evaluación \\
Evaluación de los contenidos & Evaluación de las actitudes y valores \\
Evaluación igual para todos los estudiantes & Evaluación adaptada a cada uno de los estudiantes \\
\hline
\end{tabular}

Fuente: Murillo e Hidalgo (2017, p. 6)

Un paso más allá de la concepción equitativa de la evaluación justa es la evaluación socialmente justa. No olvidemos que, como señalan Gipps y Murphy (1994), la evaluación tiene una clara e ineludible orientación social. Así, no es posible considerar la evaluación exclusivamente desde la perspectiva educativa: el acceso a la escolaridad, el currículum que se evalúa, la motivación y el afecto hacia los alumnos, los estereotipos que muchos docentes tienen hacia sus estudiantes y las expectativas docentes, interactúan con la forma en que se lleva a cabo la evaluación, y todos estos aspectos, lejos de centrarse únicamente en la escuela, tienen unas repercusiones claramente sociales.

Sin embargo, no existe consenso entre los estudiosos acerca de qué es una evaluación socialmente justa. Así, por ejemplo, Kushner (2009) considera que son cinco las características para que la evaluación sea socialmente justa: a) Proporcionar un intercambio abierto de interés entre los diferentes agentes del proceso educativo, b) Llevar a cabo la evaluación de tal forma que las desigualdades generadas puedan ser neutralizadas (promoviendo la igualdad de trato entre todos los estudiantes), c) Promover que el docente que evalúe sea imparcial, d) Publicar los resultados de la evaluación de forma abierta y pública, y e) Incrementar el reconocimiento y la responsabilidad de todos los miembros de la comunidad educativa en la evaluación.
Más interesantes son las aportaciones de Jan McArthur (2015). Esta autora defiende que una evaluación de los estudiantes socialmente justa se define entorno a dos vértices: el puramente educativo y el que juega en la promoción de una sociedad más justa. En este sentido, considera que existen cinco elementos que contribuyen a que "la evaluación sea más justa para todos aquellos que participan en ella y fomenta una mayor justicia social a través de la forma en que los estudiantes van a vivir y trabajar en la sociedad" (McArthur, 2015, p. 13). Son los siguientes:

1. Con procesos y resultados justos.

2. Equitativa, no igualitaria, que respete y valore las diferencias de cada uno de los estudiantes, ser personalizada y respetar las condiciones de origen del alumnado (nivel sociocultural y económico, raza, cultura etc.).

3. Donde coincidan las expectativas hacia los estudiantes y de los propios estudiantes, los resultados de aprendizaje y los criterios de evaluación establecidos.

4. Que considere tanto las implicaciones económicas como sociales de la evaluación y la educación.

5. Que los estudiantes estén incluidos en la toma de decisiones sobre su evaluación y su propio aprendizaje especialmente aquellos que han estado tradicionalmente excluidos. 
Con estas consideraciones teóricas, esta investigación tiene como objetivo conocer las concepciones que tienen los docentes de Educación Primaria y de Educación Secundaria sobre la evaluación socialmente justa.

\section{Metodología}

\subsection{Enfoque metodológico}

Seguramente el método más adecuado para profundizar en las concepciones de las personas, en este caso de los docentes, es la fenomenografía (Bowden y Walsh, 2000; Marton, 1986; Richardson, 1999). Este enfoque metodológico busca, por una parte, "identificar de forma cualitativa las diferentes formas en que las personas experiencian, conceptualizan, perciben y comprenden varios aspectos del fenómeno y de su mundo próximo" (Marton, 1986, p. 31) y, por otro, “...descubrir y sistematizar formas de pensamiento que sinteticen el modo en que las personas interpretan aspectos de la realidad" (Marton, 1986, p. 180).

\subsection{Participantes}

Un elemento fundamental en la investigación fenomenográ- fica es la selección de los participantes, necesitando contar con un abanico amplio de variaciones de una misma concepción que ofrezcan distintas formas de percibir e interpretar una realidad. En este estudio participaron 30 docentes de enseñanzas no universitarias. Para maximizar la variabilidad de concepciones, la selección de los participantes se hizo mediante un muestreo no probabilístico por cuotas con tres criterios de selección:

1. Nivel educativo, con 14 docentes de Educación Infantil y Primeria y 16 de Educación Secundaria.

2. Titularidad del centro, con 15 docentes que trabajan en centros de titularidad pública y 15 en centros privados (13 concertados y 2 no concertados).

3. Contexto socio-económico del centro educativo, con 15 docentes que trabajan en escuelas situadas en contextos socioeconómicos favorables y otros tantos en centros situados en contextos desafiantes. La definición del contexto se he hecho a partir del nivel socio económico del barrio donde se encuentra el centro, así como del nivel socioeconómico y cultural de las familias de los estudiantes que asisten a ese centro.

La distribución de los participantes se muestra en el cuadro 2.

Cuadro 2. Distribución de los participantes en función del contexto, del nivel educativo y de la titularidad del centro

\begin{tabular}{lcccccc}
\hline Contexto & \multicolumn{2}{c}{ Ed. Primaria } & \multicolumn{2}{c}{ Ed. Secundaria } \\
Péblico & Privado concertado & Público & Privado concertado & Privado no concertado & Total \\
Favorable & 3 & 4 & 4 & 4 & 2 & 2 \\
Total & 4 & 7 & 8 & 6 & 2 & 15 \\
\hline
\end{tabular}

Fuente: Elaboración propia.

El proceso de selección fue el siguiente. En primer lugar, se seleccionaron barrios o pueblos de la Comunidad de Madrid de alta y baja situación socioeconómica a través de indicadores de renta per cápita, tasa de paro y nivel educativo de la población, en cada zona se eligieron centros de Educación Primaria y de Educación Secundaria tanto de titularidad pública como privada. Tras contactar con dichos centros, se tuvo una breve reunión con el equipo directivo donde se informó de los propósitos y características de la investigación. Ese mismo equipo facilitó un encuentro con los posibles docentes a entrevistar en cada centro, donde se profundizó en la entrevista fenomenográfica a realizar y en la disponibilidad de cada docente. En la misma, los docentes firmaron un consentimiento de acuerdo al conocimiento del propósito de la investigación, el anonimato de las entrevistas y la fidelidad de los datos. El trabajo de campo fue desarrollado entre los meses de mayo a diciembre de 2014 y de enero a abril de 2015. En total se realizaron entre dos y tres entrevistas en cada centro. De ellos, 19 mujeres y 11 hombres, con un promedio de 13 años de experiencia docente.

\subsection{Instrumento de obtención de información}

El instrumento utilizado para la recogida de información ha sido la entrevista fenomenográfica basada en el auto-relato (Trigwell, 2000). Las entrevistas partieron de una pregunta generadora, ¿cómo es una evaluación socialmente justa?, y buscaron explícitamente no forzar las respuestas de los entrevistados. Para diseñar la entrevista, se revisaron instrumentos utilizados en investigaciones fenomenográficas análogas y éstas sirvieron para la elaboración de una entrevista con pocas preguntas generadoras orientadas a profundizar en las concepciones de los docentes participantes en el estudio.

Las entrevistas se realizaron en los centros educativos de los y las docentes, en una sala tranquila donde crear un rappport de confianza. Antes de empezar la entrevista, se garantizó la confidencialidad de los datos y concepciones de los y las participantes y obtuvimos su consentimiento informado de que las entrevistas fuesen grabadas. La duración de las entrevistas fue de entre 20 y 30 minutos cada una.

Las directrices éticas que han guiado la obtención de información con el objetivo de evitar los sesgos de la investigación fueron: a) los participantes fueron informados de los objetivos de la investigación, y se solicitó su consentimiento para ser grabados, b) se mantuvo su anonimato y c) se fue extremadamente cuidadosos de no alterar ni sus palabras ni sacarlas del contexto.

\subsection{Procedimiento y análisis de datos}

Para analizar las entrevistas, y siguiendo el proceso de análisis fenomenográfico, se ha utilizado un criterio de proximidad al discurso de los participantes. Con la ayuda del software Atlas.ti, se analizó la presencia de diferentes concepciones pertenecientes a determinadas categorías y sub-categorías en el discurso docente.

El proceso de análisis seguido fue el siguiente:

1. Identificación de las concepciones preconcebidas, que actuarán como categorías previas para el análisis. 
2. Análisis de las palabras exactas expresadas por los profesores y profesoras, dejando de lado las categorías previas del paso anterior.

3. Creación de categorías a partir de las palabras de los propios docentes en base a tres criterios: frecuencia (número de menciones), posición (lugar dentro de su discurso) y pregnancy (importancia o énfasis que le da el docente).

4. Selección de las citas más representativas de cada una de las categorías, reflejando el sentido de cada una de ellas.

5. Creación de redes de significado entre estas pre-categorías, analizándolas primero de forma individual y después de forma cruzada.

6. Establecimiento de las categorías finales, las cuales representan las distintas formas de concebir una evaluación socialmente justa.

Seguir de forma rigurosa estos pasos permite, de acuerdo a la investigación fenomenográfica, garantizar la calidad, adecuación y transferibilidad de los resultados referentes a las concepciones de los docentes hallados (ي̊kerlind, 2002; Harris, 2008).

\section{Resultados}

Las entrevistas fenomenográficas realizadas a los docentes arrojan una visión llena de matices que ayudan a profundizar en las concepciones de los profesores sobre qué es para ellos una evaluación socialmente justa.

\subsection{Evaluación para el aprendizaje}

Una de las ideas reiteradamente señalada por los docentes participantes es que para lograr una evaluación socialmente justa es necesario un cambio en la forma en que se suele concebir la evaluación de estudiantes. Muchos de los docentes señalan que la forma en que han aprendido a evaluar y ellos mismos han sido evaluados es muy tradicional, cuantitativa y con la finalidad de rendir cuentas, lo que dificulta que se planteen la evaluación de forma diferente. A pesar de ello, son conscientes del papel que juega la evaluación en la vida de sus estudiantes. Uno de los participantes indica que:

Es fundamental eliminar los prejuicios y estereotipos que se tiene de la evaluación: que el profesor no la use para clasificar o que no se separen a los estudiantes entre buenos y malos, porque eso les marca para siempre. Si estamos pensando en una sociedad mejor, esa división es una traba que marca, y si estamos pensando en un mundo mejor ese niño que tiene una tranca social obviamente va a tener otro tipo de contribución a la sociedad. [D23,pri,pu,fav,p. $2^{1}$

Como se puede observar, en esta cita emerge la idea de que la forma de evaluar clasifica y determina el desarrollo de sus estudiantes. La profesora explica que utilizar la evaluación como herramienta de clasificación, ordenación y etiquetación de los estudiantes tiene, no tan solo consecuencias negativas a nivel académico en la escuela, sino a lo largo de toda su vida. Varios de los docentes se sitúan en esta misma línea de pensamiento, considerando que la forma en que evalúen a su alumnado les influye en su vida, tanto académica como personal.

Complementando esta idea, distintos participantes señalan que es necesario un cambio en la forma de concebir la evaluación, así como ampliar la mirada en el qué y en el cómo evaluar.
Así, indican que para lograr una evaluación socialmente justa se exige un cambio radical que implica, por ejemplo, evaluar no solo los contenidos aprendidos memorísticamente valorados de forma cuantitativa, sino también, y especialmente, aquellos aspectos más cualitativos del aprendizaje que realmente muestran el progreso, avance y madurez de los estudiantes. En palabras de una maestra:

Creo que uno de los problemas para lograr una evaluación justa es que se trabajan muchos valores y actitudes, pero no se evalúan, y es fundamental que se evalúe porque entonces los alumnos verían que es importante. Por eso debería formar parte de la nota. No solo hay que evaluar lo que memorizan o el trabajo, sino también el compañerismo, la solidaridad, la reflexión y capacidad crítica... y eso sería un paso para cambiar las cosas fuera. [D30,pri,pr,fav,p.2]

Esta reflexión profundiza en que la forma tradicional en la que se vive la evaluación no es algo únicamente interiorizado por los docentes, sino también y especialmente por los estudiantes, quienes no conciben la evaluación si no es como instrumento para calificarles, ordenarles o explicitarles aquello que no hacen correctamente o no han aprendido.

Otra docente considera que muchas veces los propios docentes deberían dejar de tener miedo a cambiar su forma de evaluar y de lo que puedan opinar sus compañeros docentes, ya que, tienen que hacer uso de su libertad y autonomía en el aula. Según ella, sólo si los profesores comprenden que ellos deben compartir con sus compañeros y con la escuela las mejoras o cambios que incorporen en su evaluación de aula porque creen en ellos, sólo así, se podrá lograr una evaluación diferente:

Como el examen está tan institucionalizado en las escuelas y los padres lo apoyan, no es fácil hacer cosas diferentes. Por ejemplo, si quiero modificar la estrategia de evaluación, no los objetivos ni los contenidos, pero sí cómo voy a evaluarle, a veces recibes quejas o te cuestionan. Si puedes valorar con un trabajo, jno tiene por qué haber un examen escrito! Pero los profesores, los chicos y las familias siguen sin creérselo. Entonces tendemos a ocultar, cuando lo hacemos, a no decir que a este niño lo he valorado con un trabajo. Pues ahí estamos ya fallando porque si queremos que eso luego se extrapole a la vida y a la sociedad tenemos que hacerlo abiertamente. [D23,pri,pu,fav,p.1]

Es interesante que algún participante hace hincapié en que debería reformularse la forma en que se enseña a evaluar a los futuros docentes, ya que no se enseñan ni estrategias, ni herramientas, ni formas de concebir la evaluación desde una perspectiva alternativa. Un ejemplo:

Mi formación en evaluación fue precaria. Los docentes debemos aprender a evaluar mejor, desde la carrera hasta hacer pruebas de evaluación diferentes que midan otros aprendizajes en los chavales. [D14,sec,pu,fav,p.3]

\subsection{Evaluación equitativa}

Una segunda característica compartida por muchos de los docentes entrevistados es que la evaluación socialmente justa debe adaptarse a las características y necesidades de cada uno de los estudiantes. Asumen, así, la concepción equitativa de la evaluación frente a la igualitaria. En este sentido, los docentes con-

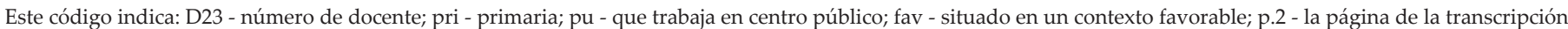
donde se encuentra la cita. 
sideran que es importante prestar más atención a los estudiantes que tienen más dificultades, aportándoles más ayuda a éstos:

Los docentes podemos proporcionar a los estudiantes que más les cuesta apoyos, aunque no haya presupuesto: por ejemplo, quédate un recreo, vente un martes por la tarde que yo te lo explico... se puede hacer, no todo el mundo lo hace, pero eso ya es una cuestión personal del profesor. [D20,sec,pr,fav,p.1]

Si un niño que se está esforzando se merece aprobar lo tiene que saber la gente, y eso es una manera también de que la gente sepa que no todos somos iguales y no todos tenemos las mismas oportunidades ya desde nacimiento, las situaciones sociales y familiares no las ha elegido nadie. [D29,sec,pu,des,p.2]

Para que una evaluación sea equitativa, los profesores consideran fundamental tener en cuenta la actitud que el propio docente tiene con los estudiantes. Así, tratarlos sin juzgarlos, sin crear falsas expectativas hacia ellos y valorándolos independientemente de la calificación que obtengan es fundamental para que la evaluación sea socialmente justa:

Lo más importante al evaluar es que vean que tratas igual a todos los estudiantes, que tanto al que saca sobresalientes como al que no hace nada pues que lo tratas igual, o que ayudas al que más lo necesita. Eso además requiere una reflexión con ellos, para que entiendan por que otros compañeros necesitan más ayuda. [D22,pri,pu,fav,p.1]

\subsection{Evaluación participativa}

Una idea más de los docentes: para que la evaluación sea socialmente justa tiene que dejar de ser responsabilidad única de los docentes y pase a ser responsabilidad compartida con los estudiantes, empoderando a estos en su propia evaluación. Los profesores consideran que solamente si los alumnos se implican verdaderamente en el proceso evaluativo formando parte del mismo se podrá lograr un cambio en la práctica evaluativa.

Una maestra de primaria destaca la importancia de compartir la responsabilidad de la evaluación con los estudiantes y también con las familias de éstos:

Es básico implicar a mis estudiantes en el diseño de la evaluación y a las familias. Yo lo hago siempre. Primero les doy el boletín a los padres para que ellos pongan nota a sus hijos del trabajo en clase, para que las familias se den cuenta de lo que es poner notas, y también les pido una observación del trabajo en casa de los chicos y su autonomía en casa y su autoconcepto. [...] Después, les doy otros a los alumnos, se hacen una propia autoevaluación donde también hacen propuesta de evaluación y luego va mi evaluación. [D27,pri,pr,fav,p.4]

Es interesante verificar que algunos docentes consideran que sólo si los estudiantes toman las riendas de su aprendizaje a través de la evaluación serán conscientes de su desarrollo y progreso y sólo así se podrá lograr que la evaluación tenga el potencial de hacerles conscientes de su aprendizaje:

No somos conscientes de lo que mejorarían los chavales si nosotros les incluyéramos en las decisiones, preguntándoles: ¿qué es para ti importante?, ¿cómo quieres que te evalúe?, ¿cómo puedo hacerlo yo para que tú mejores?, quitar los estereotipos de que el profesor manda... si tú les preguntas o les das la mínima confianza en que tu también puedes mejorar... eso mejora mucho. [D1,pri,pu,des,p.5]
Un elemento necesario, desde la perspectiva de los profesores, para dar voz a los estudiantes en su evaluación es formarles para ello. El empoderamiento de los estudiantes en la evaluación no se traduce tan solo en que los alumnos se evalúen a sí mismos y sean conscientes de su progreso en el aprendizaje, así como qué cosas mejorar, sino también que tengan la capacidad de evaluar el trabajo tanto de otros compañeros como el del docente. Esta coevaluación desarrollará en el estudiante el sentido de responsabilidad haciéndole consciente de la dificultad de evaluar justamente. Para reflejar esta idea, un profesor de primaria considera que:

No solo que el estudiante se sienta evaluado, sino que él también pueda juzgar su evaluación y que incluso pueda juzgar el trabajo de los demás y también el trabajo del profesor. [D19,pri,pr,des,p.2]

Otro elemento que los docentes destacan como importante es el uso de la asamblea en el aula. Éstos conciben que llevar a cabo asambleas es una herramienta muy útil para que los estudiantes se sientan implicados en su propio aprendizaje y aprendan que las decisiones que toman tienen consecuencias, tanto para ellos mismos como para sus compañeros. Un docente señala:

Una evaluación socialmente justa tendrá que ser por ejemplo a través de una asamblea, donde tu preguntas como se han sentido, [...] o preguntar si el alumno cree que todos han aprendido por igual, entonces él tiene que reflexionar si su amigo Jaimito ha aprendido igual que él y si eso es justo... [D28,pri,pu,des,p.4]

\subsection{Evaluación concientizadora}

En cuarto lugar, los docentes indican que es fundamental que la evaluación contribuya a concienciar a los estudiantes sobre los problemas sociales que les rodean y, con ello, comprometerles en su solución. Así, en lugar de preguntar contenidos más teóricos o desvinculados de la sociedad, los profesores y profesoras piensan que las pruebas de evaluación socialmente justa deberían incorporar textos, ejemplos o actividades que acerquen a los estudiantes a las situaciones que viven las personas con más dificultades o desventajas sociales para hacerles conscientes y así reflexionar sobre ello. Ilustrando esta idea:

En asignaturas como sociales si estás viendo por ejemplo la forma de producción empresarial hoy en día a nivel global mundial, y si de alguna manera mentalizas a tus estudiantes de que hay países subdesarrollados donde la mano de obra es más barata, evidentemente están todas las personas trabajando para que tu lleves ese jersey a buen precio, otros están trabajando a condiciones tal... [D20,sec,pr,fav,p.3]

Del mismo modo, otro docente piensa que trabajar temas de carácter social en clase y luego incorporarlos a la evaluación ayuda a que los estudiantes reflexionen sobre los mismos y se impliquen en su solución:

En lo que enseño y evalúo trato temas de injusticias, por ejemplo, el tema del medio ambiente o el ocio [...]. Usar más el autobús, ir a sitios que tienes más cerca... dejarles claro que su potencial de transformación del mundo es básico. Los políticos tienen mucho que decir, EEUU es una superpotencia sí, pero quien cambia el mundo no son los políticos sino nosotros y con nuestro día a día somos los que tenemos la capacidad de cambiar las cosas, que comprendan las consecuencias de los actos de las personas. Yo lo trabajo con debates para que vean su capacidad de transformación. [D22,pr,pu,fav,p.1] 


\subsection{Evaluación del autoconcepto y de la empatía}

Un elemento más señalado en las entrevistas fenomenográficas es la necesidad de que la evaluación ayude a los estudiantes a que reconozcan su identidad, piensen sobre ella y entiendan su situación social. Ilustrando esta concepción un profesor de Educación Primaria menciona que:

Hay que ver si el alumno sabe a qué identidad pertenece, si respeta a las otras, a las especies, al medio, si sabe lo que es injusto, si es capaz de ponerse en la piel del otro, si es capaz de tomar decisiones... [D28,pri,pu,des,p.3]

Esta identidad que menciona el docente está altamente relacionada con el autoconcepto de los estudiantes: cómo se ven, cómo se identifican, como se consideran... así como su capacidad de respetar, valorar y empatizar con otras personas. Así, desde las concepciones del profesorado, otro elemento fundamental para lograr una evaluación socialmente justa es que ésta sirva para trabajar y desarrollar un autoconcepto positivo en los estudiantes. Para algunos profesores, la evaluación puede ayudar a que los estudiantes tengan un concepto de sí mismos lo más ajustado posible a la realidad. Un ejemplo es el siguiente:

Hay que conseguir que el estudiante sea consciente de sus defectos, de sus habilidades, consciente de su fracaso, y que sea consciente de que a veces se equivoca, que puede sacar un tres o un cuatro y que a veces le va muy bien y puede sacar un 10. Yo pienso que tienes que crear consciencia en el niño, de lo que es y de lo bueno que tiene y de que el error es algo normal en el ser humano. [D18,sec,pr,des,p.2]

Uno de los profesores matiza que para trabajar el autoconcepto y la empatía es fundamental crear situaciones lo más reales posibles para conocerle mejor y ser capaces de ponerse de una forma efectiva en el lugar del otro:

Es fundamental avanzar a una evaluación más auténtica en situaciones más reales y eso ayudaría a que los estudiantes recibieran retroalimentación sobre su hacer mientras actúan en el mundo. En una situación real aparecen sentimientos, emociones, incertidumbres, miedos, que desde mi punto de vista son muy importantes. [D10,sec,pr,fav,p.2]

\subsection{Evaluación de y para la capacidad crítica y reflexiva de los estudiantes}

Por último, los docentes creen que la evaluación no solo tiene que basarse en la memorización de contenidos o procedimientos, sino que los estudiantes deberían mostrar su capacidad crítica y reflexiva. Así, una docente menciona que:

En mi evaluación quiero que me demuestren que tienen un pensamiento crítico y que aprendan a reflexionar y que no porque estén habituados a ver un comportamiento este sea bueno o correcto y es el que tienen que hacer ellos. Tienen que pensar que quieren hacer. Tienen que saber reflexionar, y para ello tienen que conocer las consecuencias de las cosas, intentar que alcancen la madurez. [D21,sec,pr,des,p.3]

Trabajar en el aula y evaluar que los estudiantes sean conscientes de los problemas existentes y reflexionen acerca de ellos es fundamental para lograr una evaluación socialmente justa:
También es fundamental que los estudiantes sean críticos, hay que luchar por ello, para que sean conscientes de las injusticias que hay en la sociedad. Pero hay que tener cuidado. Por ejemplo, ahora estamos haciendo un rastrillo solidario, y lo que se recolecta va integro a alguna asociación, y concienciar a los alumnos para ayudar esta muy bien. Lo que pasa que hemos llegado a un momento que yo estoy observando desde hace años que ahora ya lo que se trae son cosas muy sucias y en mal estado pero hay que venderlo y estamos incitando a un consumismo que no es lógico. Hay que educar también en eso, no todo vale. Si no somos críticos con esto, por ejemplo, tampoco lo son con lo que les rodea. [D23,pri,pu,fav,p.4]

Los profesores consideran que este pensamiento crítico se puede trabajar proponiendo a los estudiantes situaciones injustas o de desigualdad reales, sobre las cuales reflexionar en las pruebas de evaluación. Así, para una de las profesoras el propósito principal de la evaluación no debería ser otro que:

Es básico fomentar el pensamiento crítico, teniendo presente su contexto, mostrándoles la realidad tal cual es, su realidad. Enseñarles qué pasa cuando salimos fuera, cuando vemos que pasa en el barrio. Intento que ellos vean cuál es su contexto, y que tienen que pelear para llegar lejos. [D11,sec,pr,des,p.1]

Algunos de los docentes piensan que no solo es necesario que los estudiantes desarrollen una mentalidad crítica a través de la evaluación, sino también que la escuela sea crítica, en sí misma, a través de la cultura, de su equipo de docentes y del equipo directivo:

Además de la evaluación, la escuela si no es crítica no puede ser una escuela. Los docentes tenemos demasiada fuerza en manipular a los estudiantes, pasamos muchas horas con ellos, y hay que ver qué se dice y cómo lo dices, que vean en ti a alguien justo en la escuela y en la sociedad porque ellos lo aprenden. [D22,pri,pu,fav,p.1]

\section{Discusión y Conclusiones}

Los hallazgos de esta investigación conforman una concepción de los docentes sobre una evaluación socialmente justa multidimensional, compuesta por seis elementos complementarios. Así, se hace referencia a una evaluación que sea: para el aprendizaje (más que del aprendizaje), equitativa y diferenciada para cada estudiante, participativa, que contribuya a la toma de conciencia social, que valore la autoestima y la empatía, y que favorezca y considere la capacidad reflexiva y critica de los estudiantes.

Estos resultados son esencialmente coherentes con los hallazgos del estudio de Farrell y otros (2012). Así, los profesores entrevistados consideran que una evaluación socialmente justa es aquella que promueve la participación de los estudiantes en todo el proceso o práctica evaluativa (Ibarguren, Marín y González, 2017). Estos elementos también han sido señalados por Kushner (2009), quien considera fundamental incrementar la responsabilidad y participación de los estudiantes en el proceso evaluativo. Por su parte, McArthur (2015) destaca la importancia de reconocer el carácter marcadamente político y social de la práctica evaluativa. En el presente trabajo varios docentes también resaltan la necesidad de ser conscientes del carácter social de la evaluación en especial de su relevancia para concienciar a los estudiantes de los problemas sociales.

Los hallazgos de esta investigación también coinciden en gran medida con lo encontrado por Thomas y Madison (2010). En ambos casos se defiende que una evaluación socialmente jus- 
ta es aquella democrática, donde estudiantes y docentes trabajan conjuntamente para pensar y diseñar la evaluación (Martínez, Carrasco e Ibáñez, 2014); crítica, haciendo a los estudiantes conscientes de su propio aprendizaje y de la realidad que les rodea, y sensible con las diferencias de los estudiantes donde la evaluación se adapte a sus necesidades.

En cuanto a aspectos encontrados en este estudio que difieren de la literatura revisada, los docentes participantes también destacan que la evaluación ayude a reforzar el autoconcepto y la empatía del alumnado, no señalado en ninguno de los estudios encontrados y que ésta sirva para reforzar la conciencia de alumnos y alumnos de su propio proceso de aprendizaje, siendo capaz de destacar sus fortalezas y qué aspectos mejorar.

El análisis de las fortalezas de este trabajo lleva a destacar, en primer lugar, el uso de la fenomenografía como estrategia de aproximación a las concepciones docentes. Esta aproximación, poco utilizada en investigación educativa en España, posibilita un conocimiento profundo de las ideas implícitas sobre una evaluación socialmente justa, contribuyendo, de esta forma, a comprender mejor su práctica evaluativa y docente. La adecuada selección de los participantes, intentando que reflejen una amplia variedad de situaciones, es otro elemento sobresaliente, así como un riguroso proceso de análisis. Pero también es el uso de la metodología fenomenográfica la que proporciona alguna de sus mayores limitaciones. Así, los participantes, aunque suficientes para esta aproximación, son escasos para recoger por ejemplo la variedad de concepciones que se pueda dar en otros contextos.

De ahí que este trabajo podría complementarse con otro de corte cuantitativo, favoreciendo la generalización de los resultados e indagando acerca de los factores asociados a las diversas concepciones. Igualmente, sería necesario seguir indagando en temas tales como las concepciones de los estudiantes y las familias sobre una evaluación socialmente justa o en las concepciones en niveles universitarios. También sería importante profundizar en la relación entre las concepciones de docentes y estudiantes o entre las concepciones de los docentes y sus prácticas evaluativas.

Una evaluación no tiene sentido que sea justa pero que no sea socialmente justa ya que es indudable que la práctica evaluativa tiene repercusiones en la vida social y académica de los estudiantes. En este sentido, no puede existir verdaderamente una evaluación justa sino es socialmente justa, ya que plantear la justicia si no es ligada con la Justicia Social nos lleva inexorablemente a una concepción de la evaluación como herramienta del control social y del mantenimiento del status quo.

Una evaluación justa igualitaria parte de un planteamiento de reproducción de las desigualdades a través de las pruebas de evaluación y el uso de las mismas, mientras que una evaluación justa equitativa parte de un planteamiento de consideración de las desigualdades para revertirlas. Si estamos comprometidos en cambiar la sociedad a través de la educación es necesario que la evaluación tenga esta doble orientación: que sea justa y socialmente justa.

Urge avanzar hacia planteamientos de una evaluación socialmente justa. Las presiones de la Administración, el abuso de evaluaciones externas, la obsesión por la ordenación y clasificación de los estudiantes, entre otros elementos, hacen que, más que nunca, se necesite una evaluación que detecte las necesidades de los estudiantes, que tenga en cuenta su progreso y sus avances, que les ayude a conocer mejor su contexto y reflexionar sobre él y ser sensible con su realidad y la de los otros. Urge una evaluación acorde con las necesidades sociales actuales, que sea equitativa, sensible y que se oriente eminentemente a la Justicia Social (Murillo e Hidalgo, 2016; Murillo e Hidalgo, 2015b).

\section{Referencias}

Åkerlind, G. (2002, junio). Principles and Practice in Phenomenographic Research. Comunicación presentada en la Current Issues in Phenomenography Conference, Canberra, ACT.

Atjonen, P. (2014). Teachers' views of their assessment practice. The Curriculum Journal, 25(2), 238-259. https://doi.org/10.1080 /09585176.2013.874952

Bowden, J.A. y Walsh, E. (2000). Phenomenography. Phenomenography, 1, 154-169.

Brown, G.T.L. (2004). Teachers' conceptions of assessment: implications for policy and professional development. Assessment in Education: Principles, Policy E Practice, 11(3), 301-318. https://doi.org/10.1080/0969594042000304609

Brown, G.T.L. y Hirschfeld, G.H. (2008). Students' conceptions of assessment: Links to outcomes. Assessment in Education: Principles, Policy \& Practice, 15(1), 3-17. https://doi. org/10.1080/09695940701876003

Brown, G.T.L. y Remesal, A. (2012). Prospective Teachers' Conceptions of Assessment: A Cross-Cultural Comparison. The Spanish Journal of Psychology, 15(1), 75-89. https://doi. org/10.5209/rev_SJOP.2012.v15.n1.37286

Coll, C. y Remesal, A. (2009). Concepciones del profesorado de matemáticas acerca de las funciones de la evaluación del aprendizaje en la educación obligatoria. Infancia y Aprendizaje, 32(3), 391-404. https://doi.org/10.1174/021037009788964187

Farrell, V., Ravalli, G., Farrell, G., Kindler, P. y Hall, D. (2012, Julio). Capstone project: fair, just and accountable assessment. Comunicación presentada en la $17^{\circ}$ ACM Annual Conference on Innovation and Technology in Computer Science Education (pp. 168-173). Haifa: ACM. https://doi. org $/ 10.1145 / 2325296.2325339$

Gipps, C. V. y Murphy, P. (1994). A fair test? Assessment, achievement and equity. Nueva York, NY: Open University Press.

Harris, L. R. (2008). A phenomenographic investigation of teacher conceptions of student engagement in learning. The Australian Educational Researcher, 35(1), 57-79. https://doi. org/10.1007/BF03216875

Hidalgo, N. y Murillo, F. J. (2017). Las concepciones sobre el proceso de evaluación del aprendizaje de los estudiantes. REICE. Revista Iberoamericana de Calidad, Eficacia y Cambio en Educación, 15(1), 107-128. https://doi.org/10.15366/reice2017.15.1.007

Ibarguren, J. F. A., Marín, N. L. y González, J. M. (2017). La participación del alumnado en proyectos educativos vinculados al territorio: propuestas inclusivas en un centro escolar de secundaria. Aula abierta, 46, 91-96. https://doi.org/10.17811/ rifie.46.2017.91-96

Kushner, S. (2009). Own goals: Democracy, evaluation, and rights in millennium projects. En K.E. Ryan y J.B. Cousins (Eds.), Sage international handbook of educational evaluation (pp. 413-428). Thousand Oaks, CA: SAGE. https://doi. org/10.4135/9781452226606.n23

Lam, T. (1995). Fairness in Performance Assessment. ERIC Digest.

Martínez, P. M., Carrasco, C. J. G. e Ibáñez, R. S. (2014). Dime qué preguntas y te diré qué evalúas y enseñas. Análisis de los exámenes de ciencias sociales en tercer ciclo de Educación Primaria. Aula Abierta, 42(2), 83-89. https://doi.org/10.1016/j. aula.2014.05.002

Marton, F. (1981). Phenomenography: describing conceptions of the world around us. Instructional Science, 10, 177-200. https:// doi.org/10.1007/BF00132516

Marton, F. (1986). Phenomenography: a research approach to investigating different understandings of reality. Jour- 
nal of Thought, 21(3), 28-49. https://doi.org/10.24211/tjkte.2011.28.2.21

McArthur, J. (2015). Assessment for social justice: The role of assessment in achieving social justice. Assessment $\mathcal{E}$ Evaluation in Higher Education, 3(1), 1-15. https://doi.org/10.1080/026029 38.2015.1053429

Murillo, F.J. e Hidalgo, N. (2015a). Dime Cómo Evalúas y Te Diré Qué Sociedad Construyes. Revista Iberoamericana de Evaluación Educativa, 8(1), 5-9.

Murillo, F.J. e Hidalgo, N. (2015b). Enfoques Fundamentantes de la Evaluación de Estudiantes para la Justicia Social. Revista Iberoamericana de Evaluación Educativa, 8(1), 43-61.

Murillo, F. J., Hidalgo, N. y Flores, S. (2016). Incidencia del contexto socio-económico en las concepciones docentes sobre evaluación. Profesorado, Revista de Currículum y Formación del Profesorado, 20(3), 251-281.

Murillo, F. J. e Hidalgo, N. (2017). Students' conceptions about a fair assessment of their learning. Studies in Educational Evaluation, 53, 10-16. https://doi.org/10.1016/j.stueduc.2017.01.001

Pajares, M.F. (1992). Teachers' Beliefs and Educational Research: Cleaning Up a Messy Construct. Review of Educational Research, 62(3), 307-332. https://doi.org/10.3102/00346543062003307

Pepper, M.B. y Pathak, S. (2008). Classroom Contribution: What Do Students Perceive as Fair Assessment?. Journal of Education for Business, 83(6), 360-368. https://doi.org/10.3200/ JOEB.83.6.360-368

Pettifor, J.L. y Saklofske, D.H. (2012). Fair and ethical student assessment practices. En C. F. Webber y J. Lupart (Eds.), Leading student assessment (pp. 87-106). Dordrecht: Springer. https:// doi.org/10.1007/978-94-007-1727-5_5

Prieto, M. y Contreras, G. (2008). Las concepciones que orientan las prácticas evaluativas de los profesores: un problema a develar. Estudios Pedagógicos (Valdivia), 34(2), 245-262. https:// doi.org/10.4067/S0718-07052008000200015

Prieto, P. (2008). Creencias de los profesores sobre evaluación y efectos incidentales. Revista de Pedagogía, 29(84), 123-144. https://doi.org/10.5377/dialogos.v0i12.2193

Remesal, A. (2011). Primary and secondary teachers' conceptions of assessment: A qualitative study. Teaching and Teacher Education, 27(2), 472-482. https://doi.org/10.1016/j.tate.2010.09.017
Richardson, J.T. (1999). The concepts and methods of phenomenographic research. Review of Educational Research, 69(1), 5382. https://doi.org/10.3102/00346543069001053

Sambell, K., McDowell, L. y Brown, S. (1997). "But is it fair?": An exploratory study of student perceptions of the consequential validity of assessment. Studies in Educational Evaluation, 23(4), 349-371. https://doi.org/10.1016/S0191-491X(97)86215-3

Scott, S., Webber, C., Lupart, J., Aitken, N. y Scott, D. (2014). Fair and equitable assessment practices for all students. Assessment in Education: Principles, Policy \& Practice, 21(1), 52-70. https://doi.org/10.1080/0969594X.2013.776943

Stobart, G. (2005). Fairness in multicultural assessment systems. Assessment in Education, 12(3), 275-287. https://doi. org/10.1080/09695940500337249

Suskie, L. (2002). Fair assessment practices: Giving students equitable opportunities to demonstrate learning. Adventures in Assessment, 14, 5-10. https://doi.org/10.1007/978-1-40208905-3_8

Thomas, V.G. y Madison, A. (2010). Integration of social justice into the teaching of evaluation. American Journal of Evaluation, 31(4), 570-583. https://doi.org/10.1177/1098214010368426

Thompson, A. G. (1990). Teachers' beliefs and conceptions: A synthesis of the research. En D. A. Grouws (Ed.), Handbook of research on mathematics teaching and learning (pp. 127-146). Nueva York, NY: Macmillan. https://doi.org/10.2307/749453

Tierney, R. D., Simon, M. y Charland, J. (2011). Being Fair: Teachers' Interpretations of Principles for Standards-Based Grading. The Educational Forum, 75(3), 210-227. https://doi.org/10 $.1080 / 00131725.2011 .577669$

Trigwell, K. (2000). A phenomenographic interview on phenomenography. Phenomenography, 1, 62-82.

Van den Berg, B. (2002). Teachers' meanings regarding educational practice. Review of Educational Research, 72, 577-625.

Winterbottom, M., Brindley, S., Taber, K.S., Fisher, L.G., Finney, J. y Riga, F. (2008). Conceptions of assessment: trainee teachers' practice and values. The Curriculum Journal, 19(3), 192213. https://doi.org/10.1080/09585170802357504 